\title{
Compartmentalization and polarization studies of a Plant- Microbial Fuel Cell assembly with Cynodon dactylon
}

\author{
Kristopher Ray S. Pamintuan ${ }^{1,2, *}$, Carl Samuel A. Reyes ${ }^{1}$, and Dheya Kristalyn O. Lat ${ }^{1}$ \\ ${ }^{1}$ School of Chemical, Biological, and Materials Engineering and Sciences, Mapua University, Intramuros, Manila 1002, Philippines \\ ${ }^{2}$ Center for Renewable Bioenergy Research, Mapua University, Intramuros, Manila 1002, Philippines
}

\begin{abstract}
Plant-microbial fuel cells (PMFCs) are a class of renewable biomass energy that relies on the rhizodeposition of plants to generate power. In this study, the optimization of electrode spacing, number, and combinations were studied to maximize the power output of a soil PMFC growing Cynodon dactylon. To achieve this, compartmentalization tests were carried out as well as polarization. The anode-cathode distance was found to produce the highest voltage at 3 inches apart, wherein a smaller gap resulted to lower power, and a slight increase in the gap did not result to a loss of power. The use of multiple electrodes was also examined, and the results have shown that maximum power was obtained at inter-electrode distance of $18 \mathrm{~cm}$. Smaller gaps registered lower voltages, and larger gaps gave a sudden drop in voltage. The effect of limiting one electrode was also observed. In anode-limiting conditions, it was found that both power and power density were maximum when there are 4 cathodes corresponding to one anode. When the reverse was done, it was shown that both power and power density continuously dropped if there are multiple anodes corresponding to one cathode only. This led to the conclusion that cathode design is more crucial in PMFCs as it utilizes the rate-limiting step. The tests of using multiple paired electrodes to determine the powerpower density relationship results to a contradiction of behaviour in MFCs, wherein both power and power density increases as the electrode surface area is increased. These results are important building blocks to the goal of utilizing PMFCs in the future in larger scales with appreciable power generation.
\end{abstract}

\section{Introduction}

PMFCs are bioelectrochemical systems (BES) that utilize the plant and microbe relationship in the rhizosphere region to produce bioelectricity [1]. Its unique workings allow it to generate a continuous stream of electricity as long as the plant lives and excretes rhizodeposits. Although PMFC is a green source of energy, it is not yet economically advantageous to build a large-scale design due to its current power output. The maximum power density achieved for a PMFC is $220 \mathrm{~mW} / \mathrm{m}^{2}$ [2]. Nevertheless, the hope of commercializing the bioreactor is present.

Achieving power densities of MFCs high enough to make it economically viable is challenging due to the various internal resistances to consider such as ohmic, activation, bacterial metabolic and concentration losses [3]. A possible solution is stacking of smaller cells which maintains lower resistances [4]. Another technique is to lower the resistance of the electrodes, thereby helping to lower the overall cell resistance [5]. Ohmic losses are brought about by the resistance of the electrons and protons to flow through the electrodes and membrane, respectively. This can be lessened by reducing the distance between electrodes or by removing the membrane [6]. Concentration losses are brought about when mass transfer of chemical species such as protons and oxidants are limited. When the distance between subsequent electrodes are too close, the microbes will compete for an electrode to transfer the electrons to. Thus, limiting the available electrons readily to react with the proton and hydrogen in the cathodic region.

Concentration losses can also be brought about by the limited surface area of the electrode. A previous study found that the cathode was the limiting factor and should be optimized when engineering a PMFC [7]. Previous researchers have identified some of the factors that could affect the power output of a PMFC. In this study, with the use of Cynodon dactylon, the researchers attempted to (1) determine the optimum distances between the anode and cathode, and subsequent electrodes; (2) determine the optimum surface area to volume $(\mathrm{SA} / \mathrm{V})$ ratio of the limiting electrode (compartmentalization); and (3) identify the optimum external resistance needed to produce maximum power output (polarization). Doing so can be a step in the scale-up of such systems to deliver clean, renewable energy in an appreciable scale.

\section{Methodology}

\subsection{PMFC design}

Corresponding author: krspamintuan@mapua.edu.ph 
The batch PMFC designed measured $33 \mathrm{~cm} \times 20 \mathrm{~cm} \times 13$ $\mathrm{cm}(\mathrm{LxWxH})$ and was made of $1 / 2$ " plywood. Holes were placed at the bottom for drainage and on the sides for electrode placement. The electrodes used were graphite rods ( $2 \mathrm{~mm}$ diameter, $15 \mathrm{~cm}$ long). Each row of cathodes was placed 1 inch below the previous, with the first row 2 inches below the anodes, giving a vertical span of 4 inches total for the cathodes while the anodes are fixed in place. Each electrode on similar rows are placed $3 \mathrm{~cm}$ apart, giving a total horizontal span of $27 \mathrm{~cm}$. The PMFC assembly is shown in Figure 1.

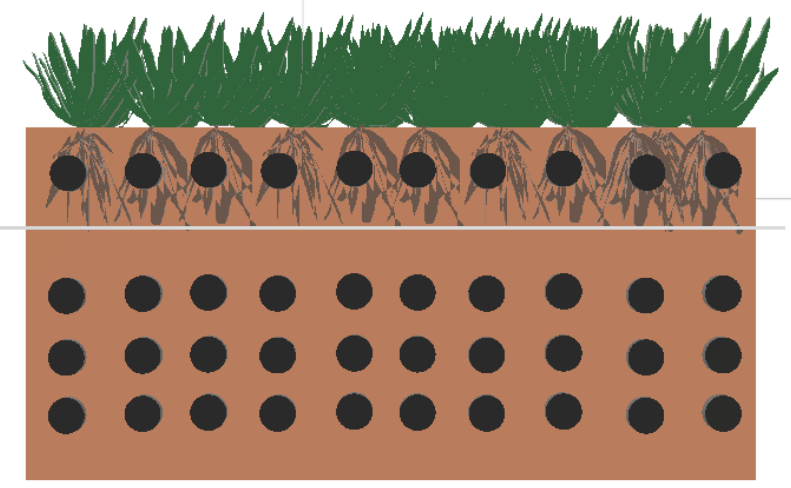

Fig. 1. PMFC assembly; from top to bottom: C. dactylon, anode array, membrane separator, cathode array

C. dactylon mats and loam garden soil were sourced from a garden supply center in Pasay City, Philippines. Similar-sized mats were placed in identical set-ups for trial replication ( 3 trials). To acclimate the plant, the mats were planted in the assembled set-up and was nurtured for 10 days, with twice daily watering. After the acclimation period, the plant has already sunk its roots below the soil surface and measurements were taken after. This period was also noted as the time it takes for the PMFC assembly to give stable, repeatable readings per day.

\subsection{Experimental set-up}

Several optimization tests were carried out as part of compartmentalization studies. First, anode-cathode vertical distance was optimized. The optimized results were then used as a basis for the electrode spacing optimization. The response of power generation on both anode-limiting and cathode-limiting conditions was also examined. This was done by pairing one anode to an increasing number of cathodes (anode-limiting) to gradually increase the surface area of one electrode while restricting the other, and vice-versa. This allows for the interpretation of which electrode is the rate-controlling area in the electrochemical reaction.

The prepared set-up also allowed for the determination of an optimum or maximum electrode surface area to reactor volume ratio $(\mathrm{A} / \mathrm{V})$, a useful metric for the enhancement of power generation of PMFCs and MFCs in general while remaining in balance with material costs. For this test, multiple paired electrodes were used in increasing numbers to determine the response of the system, measured as power generated.

\subsection{Data acquisition and analysis}

The voltage reading against a $1000-\mathrm{ohm}$ resistor was manually recorded using a multimeter. The set-up allowed the rapid measurements of different configurations by simply connecting electrodes with copper wires and alligator clips.

Voltage data were gathered for a minimum of five days under different configurations to ensure the repeatability of readings. From the raw data, current $(\mathrm{I}=\mathrm{V} / \mathrm{R})$, power $\left(\mathrm{P}=\mathrm{V}^{2} / \mathrm{R}\right)$, current density $\left(\mathrm{I}_{\mathrm{D}}=\mathrm{I} / \mathrm{A}\right)$, and power density $\left(\mathrm{P}_{\mathrm{D}}=\mathrm{P} / \mathrm{A}\right)$. Afterwards, polarization studies were performed by gradually increasing the external resistance (from 5 to $50000 \mathrm{ohms}$ ) and recording the voltage.

\section{Results and discussion}

\subsection{Electrode spacing optimization}

The measured voltage against increasing anode-cathode distance are shown in Figure 2. It is evident from the results that anode-cathode distances of 3 or 4 inches generated a higher potential difference across all testing days. Results of 3- and 4-inches distance are not significantly different across all days $(\alpha=0.05)$. These results are consistent with those previously obtained from MFCs, stating that the increase in anode-cathode distance was counter-intuitive yet effective [8].

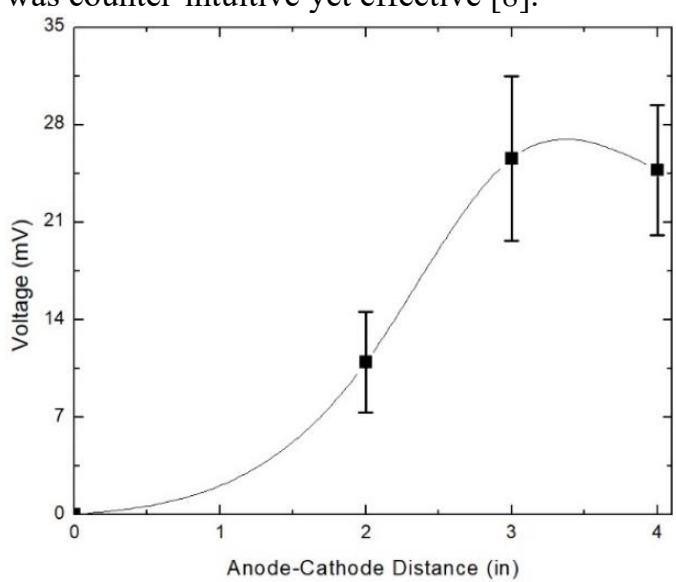

(a)

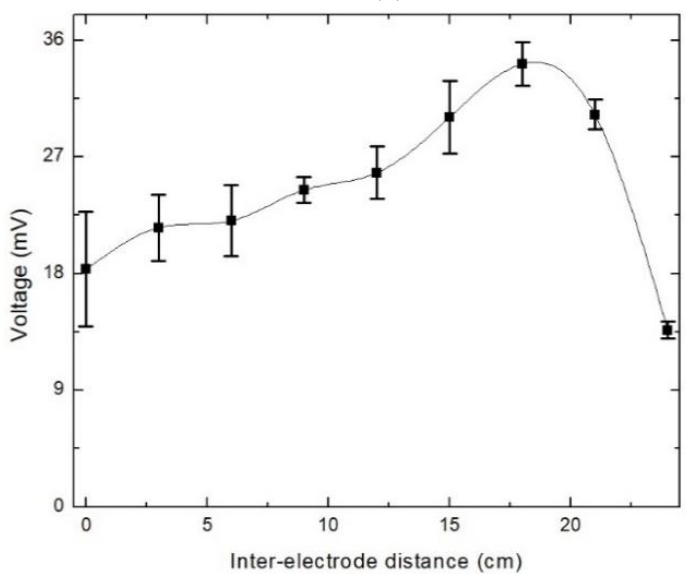

(b)

Fig. 2. (a) Anode-cathode distance optimization and (b) horizontal inter-electrode distance optimization at the 
optimized anode-cathode distance

It was previously theorized that decreasing the anodecathode distance would reduce ohmic loses and internal resistance, which in turn would increase power generation. However, the results of this study prove that an ample anode-cathode distance is necessary in a soil substrate to optimize power generation. This is due to the phenomenon called oxygen crossover wherein oxygen is introduced in the anode instead of being concentrated in the cathode, decreasing its performance. The provision of holes in the sides of the PMFC in this study allowed for more oxygen to diffuse through the cathodes, and oxygen crossover apparently is greater in the topmost row of cathodes due to its proximity with the free surface.

The results of the anode-cathode spacing optimization were used as a basis for the next part, an optimization of inter-electrode spacing. The results are shown on Figure 2. Using an anode-cathode distance of 3 inches, the measured voltage was observed to continuously increase as same electrode spacing was incrementally increased. The peak voltage was reached at an inter-electrode distance of $18 \mathrm{~cm}$, and the voltage drastically decreased after that point. This result is important in the scale-up of PMFCs as it proves that similar electrodes placed too near each other will result to increased material costs without a proportionately increased power output, while same electrodes placed too far will drastically suffer from reduced power output. The results make sense in terms of substrate resistance, wherein the soil itself offers resistance to the flow of protons produced from the anode to the cathode. Increasing the number of the same electrodes also increases the effective electrode surface area, which then increases the sites available for reaction.

\subsection{Compartmentalization effects}

The result of anode-limited compartmentalization is shown on Figure 3a. With the number of anodes fixed to one and gradually increasing the number of cathodes, it is shown that both power and power density in a PMFC proportionally increases until it reaches a maximum at 4 cathodes. Beyond that, both values considerably decreased. This shows that the optimal surface area ratio of cathode to anode is $4: 1$, giving maximum power and power density. It also shows that cathodes play a very important role in the completion of the half reaction, since it is shown to be the rate-limiting step. By increasing the surface area of the cathode, more sites are opened to match the rate of the anode half-reaction. However, if the ratio is exceeded, the additional cathodes provide more resistance that results to a decreased power output. The reported results are similar to a recently concluded study, however the optimum ratio was different as optimization was not performed in the previous study [7].

Cathode-limited conditions presented a different set of results (Figure 3b). Increasing the number of anodes while using only one cathode resulted to a rapid decrease in both power and power density. This result leads to the conclusion that the reduction at the cathode was indeed the rate-limiting step. Increasing the rate of reaction in the anode compartment while keeping the cathode compartment limited resulted to an overload of protons that cannot be immediately processed in the cathode. These results are also similar to a previous study wherein cathode-limited conditions results to a lower power generation [7]. Also, the same trend was observed in batch Microbial Fuel Cells fed with wastewater [9], leading to the speculation that the rate limiting effect of the cathodic reaction is not influenced by substrate properties (terrestrial or aquatic).

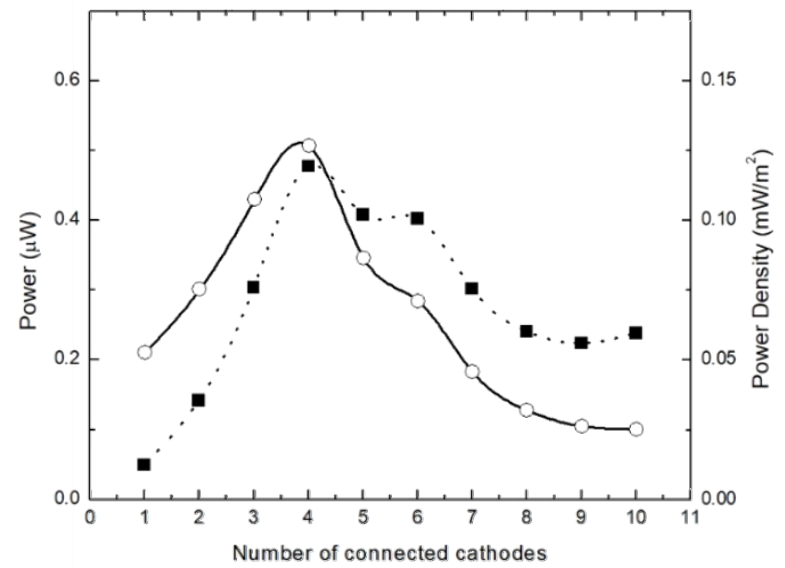

(a)

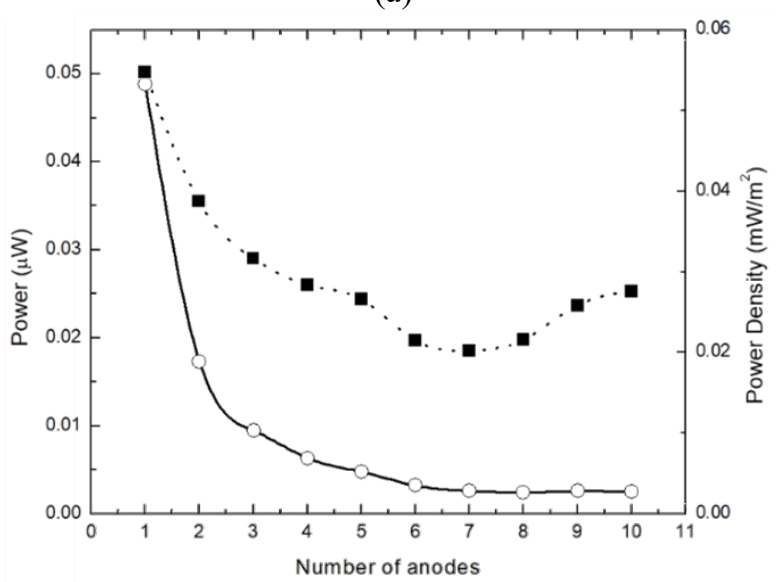

(b)

Fig. 3. Response of power ( $\square$ ) and power density (o) to the limiting conditions of (a) anode-limited and (b) cathodelimited compartmentalization

This study also reports the first attempt on maximizing power and power density by using multiple electrodes. A similar study was done in the past using MFCs [10]. The results are shown in Figure 4. Both power and power density continuously increased upon the introduction of more paired electrodes. This contradicts the results of the same test for MFCs wherein power increases while power density decreases [10]. A key difference for the discrepancy is in the source of nutrients. An MFC uses nutrients that are inherently present in the substrate (wastewater) which decreases and are not replenished in batch operation. In the case of PMFCs, the plant continuously produces rhizodeposits that act as the substrate for the electrogenic bacteria to feed on, hence both power and power density will be expected to increase as more electrodes are placed. However, this study was limited up to 9 pairs of anodes and cathodes only, and it is recommended for future 
studies to explore the limit of this phenomena. Connecting 9 pairs of anodes and cathodes correspond to an electrode surface area to volume ratio (A/V) of 0.989 $\mathrm{m}^{2} / \mathrm{m}^{3}$, the first value to be reported for soil PMFCs. The same increasing trends for both power and power density were observed for MFCs with wastewater as substrate corresponding to an electrode surface area to volume ratio of $2.34 \mathrm{~m}^{2} / \mathrm{m}^{3}$. This is significantly higher than the first reported value for PMFCs in this study. Nevertheless, the results of this study are sufficient to conclude that maximization of power and power density is achievable in PMFCs and is hoped to be a powerful driver in the scale-up of PMFCs.

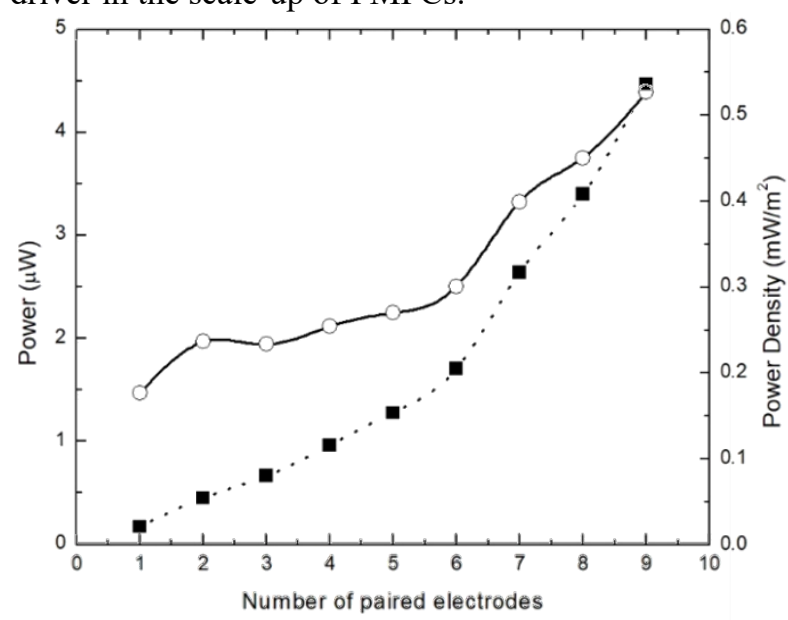

Fig. 4. Behavior of power ( $(-)$ and power density $(\circ)$ with increasing electrode surface area

\subsection{Polarization}

The generated polarization curve is shown in Figure 5. Polarization was done on a single anode-cathode pair separated by 3 inches. The resistance at the point of maximum power is registered at 3900 ohms. The high internal resistance is expected in soil set-ups due to the increased difficulty of proton and electron motion [1]. The maximum power density obtained from polarization is $0.15 \mathrm{~mW} / \mathrm{m}^{2}$ cathode area with the potential to further increase as indicated by the absence of a maxima on Figure 4.

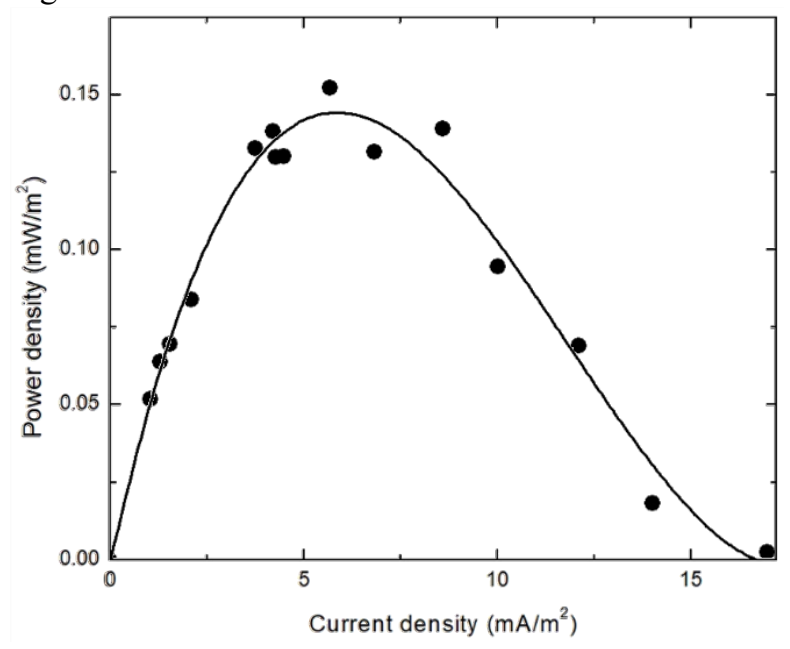

Fig. 5. Polarization curve for $C$. dactylon, single electrode
To further improve on the design, a minimization of the internal resistance is sought after. This may be in the form of a new design, or new electrode configuration. It is therefore recommended for future studies to further examine compartmentalization as part of the efforts to increase the efficiency of PMFCs.

\section{Conclusions}

This study has demonstrated the possibility of scale-up using multiple electrodes in a PMFC. Guided by the result that PMFCs are limited by cathode surface area, more efficient and larger PMFCs can be made. Cathode surface area needs to be about 4 times the anode surface area to allow more cathodic sites to facilitate a slower step, compensating for the faster oxidation in the anode. Also, the optimum anode-cathode distance as well as optimum inter-electrode distances were reported and can be used to design larger PMFCs.

The power-power density behavior of PMFCs utilizing multiple electrodes is advantageous, as it does not exhibit the reverse proportionality commonly seen in MFCs. Instead, both continually increased as the electrode surface area increased, meaning a larger PMFC has the potential to deliver larger power generation. This study reported a maximum surface area to volume ratio $(A / V)$ of $0.989 \mathrm{~m}^{2} / \mathrm{m}^{3}$, the first of its kind. The maximum number of electrodes possible was not achieved in this study, paving the way for possibly higher $\mathrm{A} / \mathrm{V}$ ratios, and as such, is recommended for further investigation.

Overall, the optimization techniques applied in this study has been successful in identifying the critical steps needed to increase the power output of PMFCs.

The authors are grateful to Mapua University for funding the publication of this study.

\section{References}

1. R. Nitisoravut and R. Regmi, "Plant microbial fuel cells: A promising biosystems engineering," Renew. Sustain. Energy Rev., vol. 76, no. March, pp. 81-89, 2017.

2. R. A. Timmers, D. P. B. T. B. Strik, H. V. M. Hamelers, and C. J. N. Buisman, "Long-term performance of a plant microbial fuel cell with Spartina anglica," Appl. Microbiol. Biotechnol., vol. 86, no. 3, pp. 973-981, 2010.

3. P. Aelterman et al., "Critical Review Microbial Fuel Cells : Methodology and Technology †," vol. 40, no. 17, pp. 5181-5192, 2006.

4. K. R. S. Pamintuan, J. A. A. Clomera, K. V. Garcia, G. R. Ravara, and E. J. G. Salamat, "Stacking of aquatic plant-microbial fuel cells growing water spinach (Ipomoea aquatica) and water lettuce (Pistia stratiotes)," in IOP Conference Series: Earth and Environmental Science, 2018, vol. 191, no. 1.

5. K. R. S. Pamintuan and K. M. Sanchez, "Power generation in a plant-microbial fuel cell assembly 
with graphite and stainless steel electrodes growing Vigna Radiata," in IOP Conference Series: Materials Science and Engineering, 2019.

6. M. Helder, D. P. Strik, H. V. M. Hamelers, and C. J. N. Buisman, "The flat-plate plant-microbial fuel cell : the effect of a new design on internal resistances The flat-plate plant-microbial fuel cell: the effect of a new design on internal resistances," Biotechnol. Biofuels, vol. 5, no. 1, p. 1, 2012.

7. N. Ueoka, N. Sese, M. Sue, A. Kouzuma, and K. Watanabe, "Sizes of Anode and Cathode Affect Electricity Generation in Rice Paddy-Field Microbial Fuel Cells," J. Sustain. Bioenergy Syst., vol. 06, no. 01, pp. 10-15, 2016.
8. S. Cheng, H. Liu, and B. Logan, "Increased Power Generation in a Continuous Flow MFC with Advective Flow through the Porous Anode and Reduced Electrode Spacing," Environ. Sci. Technol., vol. 40, no. 7, pp. 2426-2432, 2006.

9. K. R. S. Pamintuan, I. H. P. Bagumba, and Z. D. G. Domingo, "Compartmentalization studies of a deepdesign batch Microbial Fuel Cell assembly," J. Phys. Conf. Ser., vol. 1457, no. 1, 2020.

10. J. Zhang, J. Li, D. Ye, X. Zhu, Q. Liao, and B. Zhang, "Tubular bamboo charcoal for anode in microbial fuel cells," J. Power Sources, vol. 272, pp. 277-282, 2014. 